\title{
ON REGULARITY OF GENERALIZED SURFACES OF CONSTANT MEAN CURVATURE
}

\author{
BY ROBERT GULLIVER II
}

Communicated by Raoul Bott, August 7, 1970

Statement of result. A surface in a manifold is called "regular" to emphasize that it is immersed; the term "generalized surface" is used when regularity is only assumed almost everywhere.

We treat generalized surfaces of constant mean curvature in an analytic Riemannian manifold $M$ of dimension 3. To this end consider the variational problem:

$$
E[X]=D[X]+4 H V[X] \rightarrow \min
$$

where the admissible $X$ are all mappings of a domain $G \subset R^{2}$ into $M$, continuous in $\bar{G}$ and $C^{2}$ in $G$, which agree with a given admissible $X_{0}$ on $\partial G$ up to reparameterization; and where $H$ is a real constant, $D[X]$ is the Dirichlet integral of $X$

$$
D[X]=\iint_{G} g_{k l}\left(\stackrel{k}{x_{u}} x_{u}+\stackrel{k}{x_{v}} x_{v}\right) d u d v
$$

and $V[X]$ is volume of a consistently chosen region whose oriented boundary is $X-X_{0}$. A mapping which is stationary for this functional is a generalized surface of constant mean curvature $H$ in isothermal coordinates, cf. [1, pp. 107-112]. If a mapping is not only stationary but minimizing, we can say more:

TheOREM. Any mapping $X$ which minimizes $E$ among the admissible mappings defines a regular surface.

Observe that the mapping $X$ itself need not be a regular parameterization. Since the theorem can be applied in any neighborhood, it follows that a mapping which minimizes $E$ even locally must be regular. The theorem is a generalization of the work of Osserman on the Plateau problem, that is, the case $H=0, M=E^{3}[3]$.

Consequences. In the case that $M$ is a space form, examples can be constructed of generalized surfaces of constant mean curvature with

AMS 1970 subject classifications. Primary 49F22, 35B99, 49F25; Secondary 53B20, 49F10.

Key words and phrases. Constant mean curvature, branch points, Plateau problem, Riemannian manifold, calculus of variations.

Copyright @ 1971, American Mathematical Society 
singularities resembling branch points of conformal mappings. This construction was given by Courant for the case $H=0, M=E^{3}[1$, pp. $122 \mathrm{ff}]$. By the above theorem these generalized surfaces are unstable with respect to $E$ in every neighborhood of the singular point, in contrast to what is known for single-integral variational problems.

Combining the above theorem with various results on the existence of generalized surfaces as solutions to variational problems of this type improves each to a statement of existence for regular surfaces. For example, the result of Hildebrandt [2] now yields

TheOREM. Given a continuous curve $\gamma$ in the unit ball of $E^{3}$ such that some mapping with finite Dirichlet integral spans $\gamma$, and given $H$ with $|H| \leqq 1$, there exists a regular surface spanning $\gamma$ with constant mean curvature $H$.

This answers a question posed in [2], and, in similar fashion, the conjecture in $[3, \S 5.4]$ for the analytic case.

Indication of proof. The approach taken is to study the behavior of a solution to the Euler equations near a branch point, i.e. a point of nonregularity. Orthogonal projection on a certain plane is shown to be a covering map in a punctured neighborhood. Properties of nonparametric solutions are then applied toward an analysis of the selfintersections of the solution. We prove

THEOREM. There is an analytic arc of self-intersection of the solution which converges to the branch point in a well-defined direction.

Lemma. There is a neighborhood of the branch point in which either

(1) the solution intersects itself only transversally; or

(2) with appropriate choice of parameters, the solution is invariant under a rotation of the parameter domain.

The main result is then obtained in case (1) by means of a discontinuous "reparameterization" near the branch point which leaves the value of $E$ unchanged but introduces a corner. This new generalized surface cannot minimize $E$, nor, therefore, can the original solution. In case (2) the solution has an inessential branch point; the surface itself is regular.

This work can be generalized to a broad class of geometrically meaningful variational problems whose Euler equations form elliptic systems. In particular, geometric side conditions may be imposed.

Details will appear in the author's thesis. 


\section{BIBLIOGRAPHY}

1. Richard Courant, Dirichlet's principle, conformal mapping, and minimal surfaces, Interscience, New York, 1950. MR 12, 90.

2. Stefan Hildebrandt, On the Plateau problem for surfaces of constant mean curvature, Comm. Pure Appl. Math. 23 (1970), 97-114.

3. Robert Osserman, $A$ proof of the regularity everywhere of the classical solution to Plateau's problem, Ann. of Math. (2) 91 (1970), 550-569.

Stanford University, Stanford, California 94305 